\title{
Carotid Webs and Recurrent Ischemic Strokes in the Era of CT Angiography
}

\author{
P.M.C. Choi, D. Singh, A. Trivedi, DE. Qazi, D. George, J. Wong, A.M. Demchuk, M. Goyal, (DM.D. Hill, and B.K. Menon
}

\begin{abstract}
BACKGROUND AND PURPOSE: Carotid webs may cause recurrent ischemic stroke. We describe the prevalence, demographics, clinical presentation, imaging features, histopathology, and stroke risk associated with this under-recognized lesion.
\end{abstract}

MATERIALS AND METHODS: A carotid web was defined on CTA as a thin intraluminal filling defect along the posterior wall of the carotid bulb just beyond the carotid bifurcation on oblique sagittal section CTA that was seen as a septum on axial CTA. Using a prospective case series from April 2013 to April 2014, we describe the demographics, spectrum of imaging features on CTA, and histopathology of these carotid webs. From a retrospective analysis of patients at our center from May 2012 to April 2013 who had a baseline head and neck CTA followed by a brain MR imaging within 1-2 days of the CTA, we determine the period prevalence of carotid webs and the prevalence of ipsilateral stroke on imaging.

RESULTS: In the prospective series, the mean age was 50 years (range, 41-55 years); 5/7 patients were women. Recurrent stroke was seen in $5 / 7(71.4 \%)$ patients with the carotid web; time to recurrence ranged from 1 to 97 months. Histopathology suggested a high probability of fibromuscular dysplasia. In the retrospective series, carotid webs were seen in 7/576 patients for a hospital-based-period prevalence of $1.2 \%(95 \% \mathrm{Cl}, 0.4 \%-2.5 \%)$. Two of these 7 patients had acute stroke in the vascular territory of the carotid web.

CONCLUSIONS: A carotid web may contribute to recurrent ischemic stroke in patients with no other determined stroke mechanism. Intimal variant fibromuscular dysplasia is the pathologic diagnosis in most cases. The prevalence of carotid web is low, while the optimal management strategy remains unknown.

ABBREVIATIONS: $A$ SA $=$ acetylsalicylic acid; $F M D=$ fibromuscular dysplasia

$\mathbf{F}$ or $>50$ years, a shelf-like projection within the lumen of the carotid bulb on vascular imaging has been referred to as a web in the carotid artery. Momose and $\mathrm{New}^{1}$ first used the term "web" in 1973 to describe this entity on carotid angiography in 4 patients in a series of 7000 patients during 8 years at Massachusetts General Hospital. The authors distinguished this entity from fibromuscular dysplasia (FMD) and postulated that carotid webs were developmental in origin. In 1977, Osborn and Anderson ${ }^{2}$ described a patient with an isolated, smooth, well-defined web at

Received January 2, 2015; accepted after revision March 19

From the Calgary Stroke Program, Department of Clinical Neuroscience (P.M.C.C., D.S., A.T., E.Q., J.W., A.M.D., M.G., M.D.H., B.K.M.), and Departments of Pathology and Laboratory Medicine (D.G.), Radiology (J.W., A.M.D., M.G., M.D.H., B.K.M.), and Medicine and Community Health Science (M.D.H.), University of Calgary, Calgary, Alberta, Canada; and Hotchkiss Brain Institute (A.M.D., M.G., M.D.H., B.K.M.), Calgary, Alberta, Canada.

Please address correspondence to Bijoy K. Menon, MD, 1079 A, 29th St NW, Calgary, AB, Canada T3H4J2; e-mail: Bijoy.Menon@Albertahealthservices.ca

三 Indicates article with supplemental on-line tables.

http://dx.doi.org/10.3174/ajnr.A4431 the origin of the internal carotid artery on conventional angiography in a case series of patients with FMD. The radiologic features were very similar to those in a histologically proved case of FMD of the carotid artery described by Rainer et $\mathrm{al}^{3}$ in 1968.

Apart from 1 isolated case report, ${ }^{4}$ in which "intraluminal web" was used to described multiple membranous strands within the carotid artery lumen, the terms "carotid web" and "pseudovalvular fold" have been used to refer to an imaging entity that appears as a small, shelf-like linear filling defect projecting superiorly into the arterial lumen and arising from the posterior aspect of the proximal internal carotid artery on conventional angiography. ${ }^{5}$ Here, we report 12 cases of carotid web from a combined prospective and retrospective series. We describe in detail the prevalence, demographics, clinical presentation, imaging features, histopathology, and stroke risk associated with carotid webs.

\section{MATERIALS AND METHODS}

All patients included in this study are part of the Calgary CT angiography data base, an ongoing imaging registry of patients 


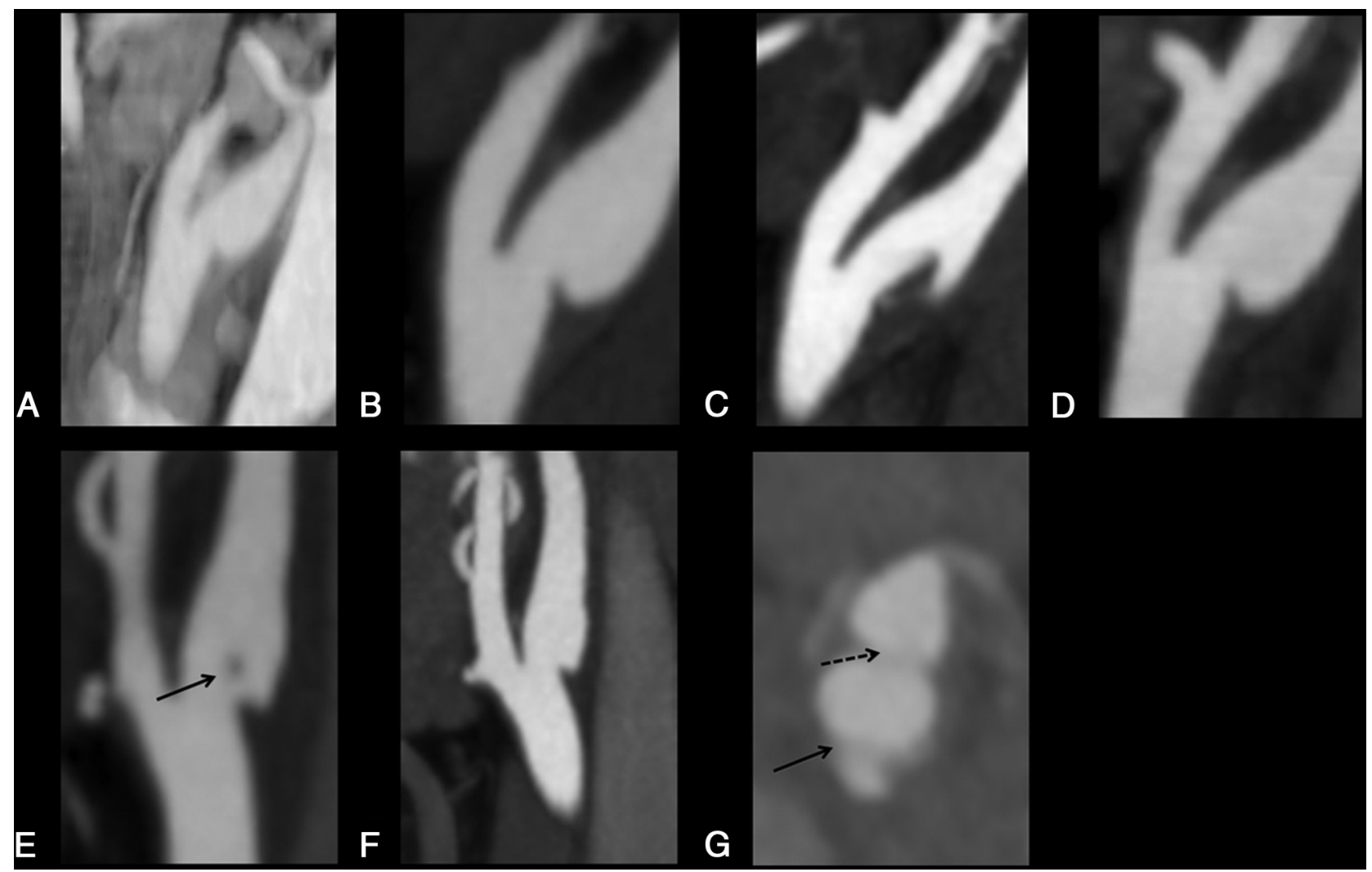

FIG 1. Sagittal and axial images of carotid webs. The top panel shows serial sagittal-view CTAs in patient A. $A$ and $B$, Carotid webs 8 years apart. $C$, Changing morphology of the right carotid web within days with possible thrombus formation in the setting of dual antiplatelet therapy. $D$, A return to baseline morphology with the use of unfractionated heparin. The bottom panel shows serial CTAs in patient B. E, A carotid web with possible thrombus in the lumen (arrow). F, The same carotid web 1 month later with no thrombus. G, An axial-view CTA with the same carotid web appearing as a septum (arrow). The broken arrow indicates the carotid bifurcation.

with acute ischemic stroke. Details of this registry have been previously described. ${ }^{6}$

\section{Prospective Case Series}

From April 2013, when we first identified a patient with a carotid web and ipsilesional ischemic stroke on head and neck CTA, we have identified 7 such patients prospectively until April 2014. The diagnosis of stroke was based on clinical grounds, supported by positive MR imaging findings in all cases. We defined a carotid web on CTA as follows: a thin intraluminal filling defect along the posterior wall of the carotid bulb just beyond the carotid bifurcation on oblique sagittal section CTA and seen as a septum on axial CTA (Fig $1 A-F$ ). We collected information on demographics, clinical characteristics, drugs, and treatment offered at baseline and during hospital stays in these patients. Stroke severity was assessed by using the NIHSS at baseline, at discharge, and at 90 days. Functional status was assessed by using the mRS at similar time points. Four of 7 patients underwent carotid endarterectomy at the discretion of the treating physician. The endarterectomy specimens were sectioned in the axial plane, fixed in formalin, and embedded in paraffin. Sections were stained for light microscopy with the following: hematoxylin-eosin, Masson trichrome, and Musto (elastin).

\section{Retrospective Series}

We also identified consecutive patients who presented to our center from May 2012 to April 2013 and had a baseline head and neck
CTA followed by a follow-up brain MR imaging at 24-48 hours. Baseline neck CTA was assessed for the presence or absence of a carotid web. Follow-up brain MR imaging (DWI and/or FLAIR) was assessed for the presence of any ischemic stroke. The side of the carotid web (left versus right) and ischemic stroke (left versus right versus posterior) was identified on baseline CTA. All images were read by consensus by 3 experienced raters (P.M.C.C., D.S., and A.T.). The readers were blinded to baseline scans while reading follow-up MR imaging.

We describe, by using standard summary statistics, the demographics and clinical characteristics of all patients (in both prospective and retrospective series). The Conjoint Health Research Board of the University of Calgary has approved the use of the CTA data base for imaging-related studies.

\section{RESULTS}

\section{Demographics}

During a 12-month period, ipsilateral carotid webs were identified prospectively in 7 patients with acute ischemic stroke (Online Table 1). The mean age of these patients was 50 years (range, 41-55 years); 5 of the 7 patients were women. Five of the 7 patients had recurrent stroke in the ipsilateral vascular territory; 1 of those 5 had 4 recurrent strokes in the same vascular territory. These patients, in total, experienced 14 ischemic strokes during 8.5 years with time to recurrence ranging from 1 to 97 months. In 2 patients, thrombus-like material was seen at the site of the carotid web, with changing morphology on serial CTAs, despite concur- 


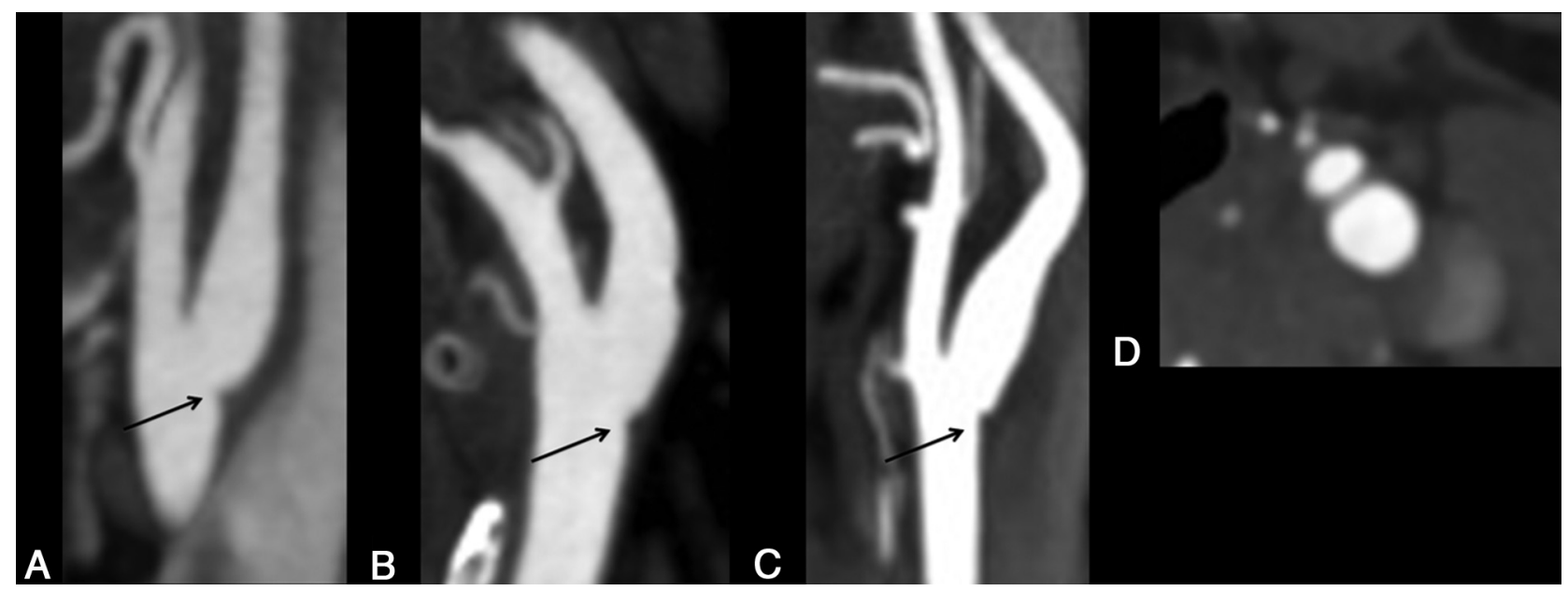

FIG 2. Small protruding lesions that are distinct from carotid webs in 3 different patients on sagittal and axial CTA (A-C, arrow). Axial CTA shows no septum with this lesion, unlike in carotid webs.

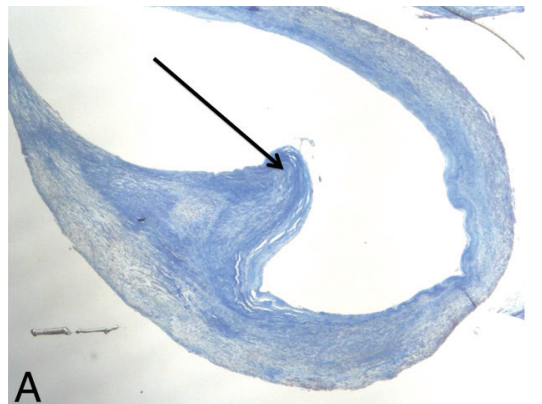

A

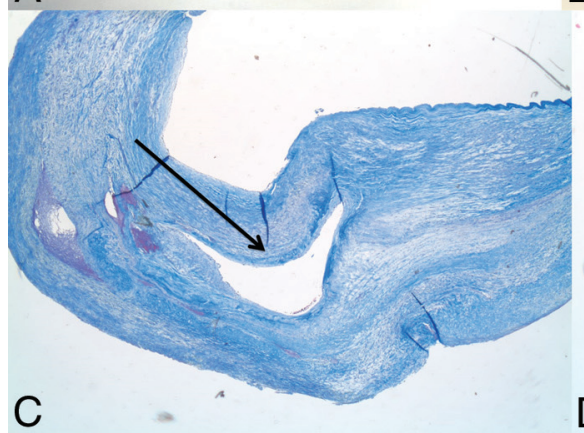

B
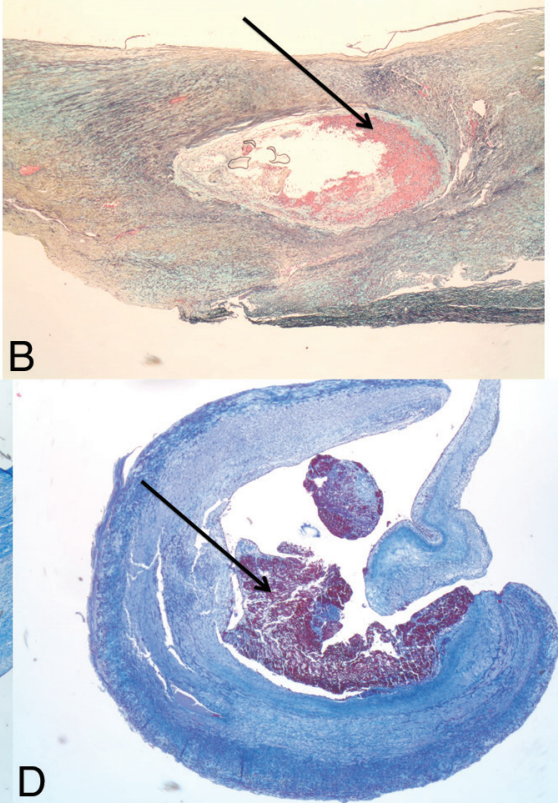

FIG 3. Figures show the histopathology of carotid webs. $A$, A shelf-like projection of abnormal intimal fibrous tissue. $B$, Focal hemorrhagic dissection with early organization. $C$, Fibrous intimal thickening with focal dissection into the fibrotic intima. $D$, Focal fibrous intimal thickening with adherent thrombus.

rent antiplatelet therapy. Four of 5 patients with recurrent strokes underwent carotid endarterectomy and remained stroke-free at last follow-up (range, 3-7 months). All our patients had no other identified cause of stroke after standard work-up. Patient characteristics and other details are described in On-line Table 1.

\section{Prevalence}

Estimating the period prevalence of the carotid web would need a population-based imaging study. This was not practical. We therefore attempted to study period prevalence in a hospitalbased sample of convenience. CTAs from 576 patients who presented to our center from May 2012 to April 2013 with suspected stroke were reviewed. These patients had a brain MR imaging performed within 1-2 days of presenting symptoms. Seven carotid webs were identified in 576 individual patients: 4 on the left and 3 on the right (On-line Table 2). Thus, the prevalence of carotid webs in a hospital-based series is estimated at $1.2 \%$ (95\% CI, $0.4 \%-2.5 \%$ ). The mean age of these patients was 63 years (range, $47-78$ years). Four of the 7 patients were women. Two of 7 patients had acute stroke in the vascular territory of the carotid web.

In our retrospective series, we also identified patients with single, small, protruding lesions in the proximal ICA. In contrast to carotid webs, these lesions were smaller and not seen on the axial sections (Fig 2). None of the patients in our prospective series had this appearance on CTA. These small protruding lesions were identified in 14/576 patients: 7 on the left and 7 on the right (On-line Table 2) for an estimated prevalence of $2.43 \%$ (95\% CI, 1.3\%-4.0\%). The mean age of these patients was also 63 years (range, 39-90 years). Six of the 14 patients were woman. Three of 14 patients had acute stroke in the vascular territory of the small protruding lesion.

\section{Pathology}

Each of the endarterectomy specimens was characterized by foci of marked fibroelastic thickening of the intima. None contained the necrotic, cholesterol-rich core of a classic atheroma. In 1 specimen, there was a shelf of intimal fibrous tissue that projected into the lumen (Fig $3 A$ ). In 2 cases, the fibrous intimal cushion was split by a dissection: One showed an organizing hemorrhage within (Fig 3B), while another formed an open endothelial-lined cavity (Fig $3 C$ ). Specimens from another case contained a thick fibrous intimal cushion with a recent mural thrombus (Fig 3D). As is characteristic of endarterectomy specimens, each specimen had an attenuated rim of elastin- 

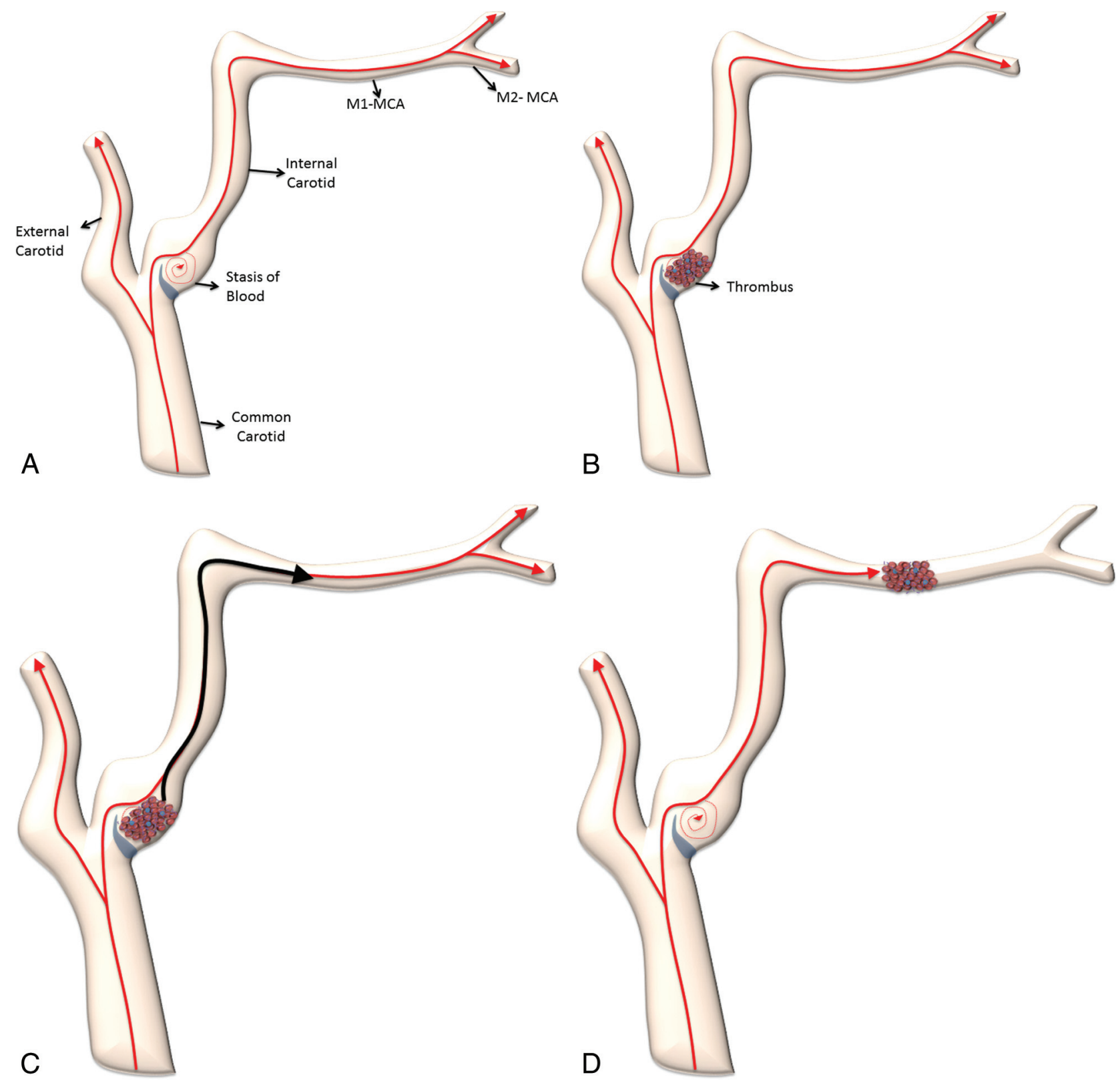

FIG 4. Animated figures depict thrombogenicity in the internal carotid artery due to the presence of a carotid web. A, Stasis of blood flow developing distal to the carotid web results in thrombus formation $(B)$. This thrombus, when of sufficient size, dislodges and embolizes intracranially $(C$ and $D)$.

rich medial tissue, but in none was a full-thickness medial section available.

Below we describe the clinical history and imaging findings of 2 patients in detail.

\section{Patient A}

A 54-year-old man (patient 1, On-line Table 1) was found slumped on the sofa with left-sided hemiparesis. He was last seen healthy 45 minutes prior. Medical history was unremarkable apart from a remote episode of transient left-arm weakness and numbness. On arrival, he had moderate left hemiplegia, neglect, and sensory loss; the National Institutes of Health Stroke Scale score was 11. Noncontrast brain CT showed early ischemic changes in the lentiform nucleus and the lateral MCA territory (ASPECTS $=8)$. CT angiography showed a mid-M1 occlusion on the right. Intravenous tPA was given followed by intra-arterial tPA combined with primary thrombus angioplasty. At 4 hours poststroke, his NIHSS score was zero. A shelf-like narrowing at the proximal right internal carotid artery with no ulceration was noted both on CTA (Fig 1A) and at the time of the catheter angiography. Investigations were negative for diabetes or hyperlipidemia; 24-hour Holter monitoring and transthoracic echocardiogram findings were normal. The patient made a full recovery and was treated with dual antiplatelet therapy for 3 months, followed by acetylsalicylic acid (ASA) and simvastatin, $40 \mathrm{mg}$ long term.

Eight years later, the patient woke up with acute mild left hemiparesis with an NIHSS score of 4 . He had stopped ASA 7 days prior for excision of a basal cell carcinoma on his left ear and 
restarted ASA the night before his presentation. NCCT showed no new ischemic change; CTA showed a new proximal right M1 occlusion and the same shelf-like narrowing in the right ICA (Fig $1 B)$. Because he was last seen healthy $>12$ hours prior, no thrombolysis was administered. He received a loading dose of clopidogrel. MR imaging showed a new striatocapsular infarct with persistent occlusion of the M1 MCA 2 days later. Repeat CTA 4 days later showed changing morphology of the right carotid web (shelf-like narrowing), suggestive of thrombus formation in the setting of dual antiplatelet therapy (Fig 1C). Transcranial Doppler studies on 2 occasions were negative for microembolic signals. Clopidogrel was stopped, and he was treated with intravenous unfractionated heparin and ASA for 3 days with resolution of the thrombus on repeat CTA 5 days later (Fig 1D). Carotid endarterectomy was performed 12 days poststroke. Pathology from carotid endarterectomy showed a focal shelf-like projection of the fibrotic intima into the lumen without any changes typical of atherosclerosis (Fig 3A).

\section{Patient B}

A 59-year-old woman (patient 2, On-line Table 1) presented with sudden onset of left hemiparesis. There was a history of recurrent right hemispheric strokes 2 and 7 years prior for which she was treated with ASA and had no residual symptoms. On arrival, she had an NIHSS score of 11 . NCCT of the brain showed an old right basal ganglia infarct with early ischemic changes in the right insula and right M2 region; her ASPECTS was 8. CTA revealed a right M1 MCA occlusion and a carotid web just distal to the ICA bifurcation with probable thrombus-like material attached (Fig 1E). She received IV tPA followed by endovascular therapy. Recanalization (TICI 2b/3) was achieved postprocedure with an NIHSS score of 2. She was discharged on dual antiplatelet therapy.

One month later, the patient was found hemiplegic on the left side. On arrival, her NIHSS score was 12. NCCT showed early ischemic changes in the right temporal cortex; CTA again showed a right $\mathrm{M} 1$ occlusion. The carotid web in the right ICA was again noted (Fig $1 F$ ). She was treated with endovascular therapy, and the right MCA recanalized. Her NIHSS score was 1 postprocedure. This time, right carotid endarterectomy was performed 8 days poststroke, with pathology showing fibrous intimal thickening with focal hemorrhagic dissection (Fig 3B). There was no history to suggest medication noncompliance before her recent stroke.

\section{DISCUSSION}

A carotid web, as an imaging entity, can be observed on both oblique sagittal reformats and axial sections of CTA. An operational definition is a thin intraluminal filling defect along the posterior wall of the carotid bulb in oblique sagittal reformats and, most important, a septum evident on axial section CTA. We demonstrate that carotid webs, though rare, are associated with recurrent ipsilateral strokes, possibly because they serve as a nidus for thrombus formation. CTA provides a reliable representation of gross structures protruding into the ICA lumen and should therefore be the imaging method of choice in noninvasive detection of carotid webs. Carotid webs may be detected by carotid sonography, but sensitivity is limited by their size and non-flow-limiting nature. ${ }^{5,7}$ With a few exceptions, ${ }^{5,7-10}$ previous reports of carotid web descriptions relied on conventional angiography. ${ }^{1,3,11-15}$ The MRA appearance of the carotid web is expected to be similar to that on CTA, though this needs to be studied.

Endarterectomy performed for atherosclerotic carotid stenosis typically produces a specimen with a classic atheroma, characterized by a large necrotic lipid-rich core covered by a fibrous cap. A thin rim of atrophic media is often adherent to the outer aspect of the atheroma. The endarterectomy specimen in each of our cases, in contrast, was characterized by thick fibrous intimal cushions associated with a shelf in 1 (Fig $3 A$ ), dissections in 2 (Fig $3 B,-C$ ), and a mural thrombus in 1 (Fig $3 D$ ). Fibrous intimal thickening noted in our specimens may be a feature of the intimal variant of fibromuscular dysplasia in the internal carotid artery. ${ }^{12,13,15,16}$ The intimal shelf observed in 1 of our patients has been previously described as an intimal variant of FMD. ${ }^{16}$ It is also possible that the various forms of asymmetric fibrous intimal cushions observed in the other 3 patients in our study represent part of the spectrum of intimal FMD in the internal carotid artery. Our ability to comment on medial variants of FMD is hampered by a lack of full-thickness medial sampling in our endarterectomy specimens. Medial fibroplasia, ${ }^{3}$ medial hyperplasia, ${ }^{11}$ and medial dysplasia ${ }^{10}$ have all been reported previously, the first 2 from fullsegment resection of the carotid artery, while the latter was said to be from an endarterectomy specimen. Carotid webs containing fibroelastic-with-muscular tissue ${ }^{14}$ and fibroelastic tissue with areas of myxoid degeneration ${ }^{8}$ have also been reported. Most interesting, none of our cases had an abnormal enlargement of the carotid bulb (megacarotid) previously described with carotid webs. ${ }^{5,17}$ None of our patients had evidence of FMD in any other vascular bed.

It is likely that the carotid web played an integral part in the stroke mechanism in all our patients. Even a short period of antiplatelet discontinuation appeared to be associated with increased thrombogenicity. In 1 patient, thrombus evolved during antiplatelet therapy, eventually responding to anticoagulant therapy. Regardless of the actual pathology, we postulate that it is the morphology rather than the histology of the web that is important in determining thrombogenicity. We postulate the existence of turbulence and stasis in a cul-de-sac upstream to the web that could potentially create a thrombogenic milieu (Fig 4). Like the left atrial appendage, this thrombogenic milieu may potentially respond best to anticoagulants. It is possible that unlike carotid webs, the small protruding lesions we describe in our retrospective series (Fig 2) may not be as thrombogenic. Although a recent case series seems to suggest that these lesions may have the same pathology as carotid webs, we cannot confirm this. ${ }^{5}$ Moreover, 3 of 14 patients with these lesions in our retrospective series had ipsilateral stroke. None of the patients in our prospective case series who had recurrent strokes had this imaging appearance though.

\section{CONCLUSIONS}

The carotid web may be an important cause of ischemic stroke in patients with otherwise no determined mechanism of stroke and may present a high risk of recurrent stroke. Intimal variant FMD may be the pathologic diagnosis in most cases. The prevalence of a carotid web in both the general and stroke population is low, and 
the optimal management strategy remains unknown. Future effort should be directed at further understanding the epidemiology, etiology, and factors associated with thrombogenicity of these lesions.

\section{ACKNOWLEDGMENTS}

We acknowledge the Calgary Stroke Program fellows, nurses, and staff including the research office for helping with the conduct, data collection, and execution of the study. We acknowledge the Seaman Family MR Research Centre and the Hotchkiss Brain Institute for their support.

Disclosures: Andrew M. Demchuk-UNRELATED: Payment for Lectures (including service on Speakers Bureaus): Covidien (modest). Mayank Goyal-UNRELATED: Consultancy: Covidien/ev3, Comments: for teaching engagements; Grants/Grants Pending: Covidien/ev3, ${ }^{*}$ Comments: partial funding for the Evaluation Study of Congestive Heart Failure and Pulmonary Artery Catheterization Effectiveness trial; Payment for Lectures (including service on Speakers Bureaus): Covidien/ev3, Comments: for teaching, designing trials. Michael D. Hill—UNRELATED: Stock/Stock Options: Calgary Scientific Inc, Comments: Stock ownership. Calgary Scientific Inc is an image software company. *Money paid to the institution.

\section{REFERENCES}

1. Momose KJ, New PF. Non-atheromatous stenosis and occlusion of the internal carotid artery and its main branches. Am J Roentgenol Radium Ther Nucl Med 1973;118:550-66 CrossRef Medline

2. Osborn AG, Anderson RE. Angiographic spectrum of cervical and intracranial fibromuscular dysplasia. Stroke 1977;8:617-26 CrossRef Medline

3. Rainer WG, Cramer GG, Newby JP, et al. Fibromuscular hyperplasia of the carotid artery causing positional cerebral ischemia. Ann Surg 1968;167:444-46 CrossRef Medline

4. Karlson KJ, Wolf B, Neptune WB. Symptomatic carotid stenosis secondary to an intraluminal web: a case report. Vasc Endovascular Surg 1987;21:422-26 CrossRef

5. Joux J, Chausson N, Jeannin S, et al. Carotid-bulb atypical fibromus- cular dysplasia in young Afro-Caribbean patients with stroke. Stroke 2014;45:3711-13 CrossRef Medline

6. Menon BK, Smith EE, Modi J, et al. Regional leptomeningeal score on CT angiography predicts clinical and imaging outcomes in patients with acute anterior circulation occlusions. AJNR Am J Neuroradiol 2011;32:1640 - 45 CrossRef Medline

7. Kliewer MA, Carroll BA. Ultrasound case of the day: internal carotid artery web (atypical fibromuscular dysplasia). Radiographics 1991; 11:504-05 CrossRef Medline

8. Gironell A, Martí-Fàbregas J, de Juan-Delago M, et al. Carotid pseudo-valvular fold: a probable cause of ischaemic stroke. J Neurol 1995;242:351-53 CrossRef Medline

9. Cordoliani MA, Lucas C, Durieu I, et al. Carotid pseudovalvular fold: a case diagnosed by angiography and helical cervical tomography. Stroke 1996;27:1010 Medline

10. Perren F, Urbano L, Rossetti AO, et al. Ultrasound image of a single symptomatic carotid stenosis disclosed as fibromuscular dysplasia. Neurology 2004;62:1023-24 CrossRef Medline

11. Gee W, Burton R, Stoney RJ. Atypical fibromuscular hyperplasia involving the carotid artery. Ann Surg 1974;180:136-38 CrossRef Medline

12. So EL, Toole JF, Moody DM, et al. Cerebral embolism from septal fibromuscular dysplasia of the common carotid artery. Ann Neurol 1979;6:75-78 CrossRef Medline

13. Wirth FP, Miller WA, Russell AP. Atypical fibromuscular hyperplasia. Report of two cases. J Neurosurg 1981;54:685-89 CrossRef Medline

14. McNamara MF. The carotid web: a developmental anomaly of the brachiocephalic system. Ann Vasc Surg 1987;1:595-97 CrossRef Medline

15. Morgenlander JC, Goldstein LB. Recurrent transient ischemic attacks and stroke in association with an internal carotid artery web. Stroke 1991;22:94-98 CrossRef Medline

16. Touzé E, Oppenheim C, Trystram D, et al. Fibromuscular dysplasia of cervical and intracranial arteries. Int J Stroke 2010;5:296-305 CrossRef Medline

17. Kubis N, Von Langsdorff D, Petitjean C, et al. Thrombotic carotid megabulb: fibromuscular dysplasia, septae, and ischemic stroke. Neurology 1999;52:883-86 CrossRef Medline 